\title{
Modeling of Disk-Star Interaction: Different Regimes of Accretion and Variability
}

\author{
Marina M. Romanova, Akshay K. Kulkarni \\ Dept. of Astronomy, Cornell University, Ithaca, NY 14853 \\ akshay@astro.cornell.edu, romanova@astro.cornell.edu \\ Min Long \\ Dept. of Physics, Center for Theoretical Astrophysics, University of Illinois at Urbana-Champaign, \\ Urbana IL, 61801 \\ longQastro.cornell.edu \\ Richard V.E. Lovelace \\ Dept. of Astronomy and Dept. of Applied and Eng. Physics, Cornell University, Ithaca, NY 14853 \\ lovelace@astro.cornell.edu
}

\begin{abstract}
The appearance and time variability of accreting millisecond X-ray pulsars (hereafter AMXPs, e.g. Wijnands \& van der Klis 1998) depends strongly on the accretion rate, the effective viscosity and the effective magnetic diffusivity of the disk-magnetosphere boundary. The accretion rate is the main parameter which determines the location of the magnetospheric radius of the star for a given stellar magnetic field. We introduce a classification of accreting neutron stars as a function of the accretion rate and show the corresponding stages obtained from our global 3D magnetohydrodynamic (MHD) simulations and from our axisymmetric MHD simulations. We discuss the expected variability features in each stage of accretion, both periodic and quasi-periodic (QPOs). We conclude that the periodicity may be suppressed at both very high and very low accretion rates. In addition the periodicity may disappear when ordered funnel flow accretion is replaced by disordered accretion through the interchange instability.
\end{abstract}

Subject headings: accretion, accretion discs; instabilities; MHD; stars: oscillations; stars: magnetic fields

\section{Different Regimes of Accretion}

A. BOUNDARY LAYER (BL). If the accretion rate is sufficiently large that $r_{m}<R_{*}$, then the star's magnetic field is completely buried by the accreting matter (e.g. Cumming, Zweibel, Bildsten 2001; 

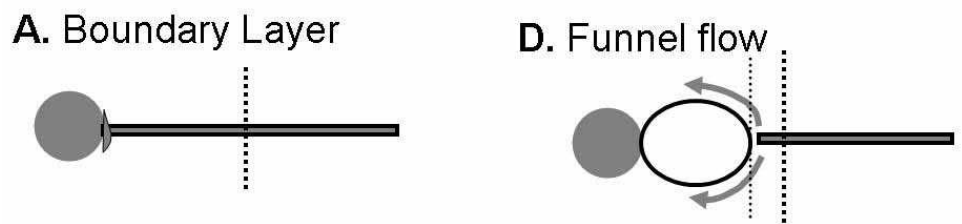

B. Magnetic BL

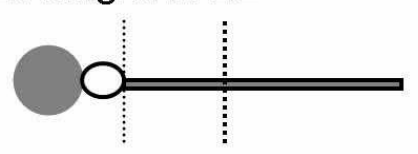

E. "Propeller"
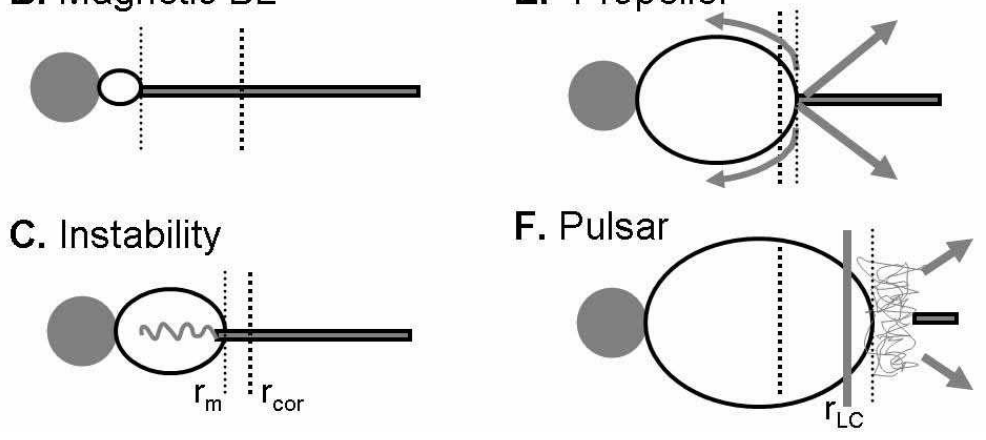

Fig. 1.- Main regimes of accretion to a rotating neutron star which occur for different accretion rates. Cases $\mathrm{A}$ to $\mathrm{F}$ show different regimes when the accretion rate gradually decreases. A is the Boundary Layer regime where the magnetic field is completely buried or screened, and matter accretes directly to the surface of the star; B is the Magnetic Boundary Layer regime where the magnetosphere is small but may influence the dynamics of the matter flow; Regimes C and D are where the magnetosphere is large enough to channel the matter flow; however, accretion may also occur through instabilities (case C); Regime E is the Propeller stage. Regime F is the radio-pulsar stage. 
Lovelace et al. 2005), and matter accretes to the star directly through a boundary layer, which is expected to be approximately axisymmetric because there is no channeling by the magnetic field. In this case, high-frequency QPOs may be associated with inhomogeneities at the boundary between inner radius of the disk and the star. Axisymmetric hydrodynamic simulations of accretion through the BL have been recently performed by Fisker \& Balsara (2005). Additional factors such as radiative pressure in the inner regions of the disk around NS may also determine the characteristic radius in the disk and corresponding high-frequency QPOs (e.g., Miller, Lamb, \& Psaltis 1998; Miller \& Lamb 2001).

B. MAGNETIC BOUNDARY LAYER (MBL). At lower accretion rates, the star's magnetic field is only partially buried, so that it influences matter flow around the star. We will draw an approximate line between MBL case and case of larger magnetospheres at $r_{m}=2 R_{*}$, so that the MBL cases correspond to $R_{*}<r_{m}<2 R_{*}$. Even in this range there are a number of possible cases with different observational properties (Romanova \& Kulkarni 2008).

MBL with No Magnetosphere. If the magnetospheric radius is very close to the stellar radius, then it cannot channel matter to the poles. However, some magnetic flux threads the inner regions of the disk and thus may influence the dynamics of the disk (e.g., Warner \& Woudt 2002). The authors suggested that the inner region of the disk may form an equatorial belt, which may be responsible for the QPOs in dwarf novae, a sub-class of cataclysmic variables. Such MBLs were proposed theoretically and should be investigated in greater detail in the future. First exploratory simulations have been done by Romanova \& Kulkarni (2008).

MBL with Small Magnetosphere. We investigated accretion to a star with a small magnetosphere in global 3D MHD simulations (Romanova \& Kulkarni 2008). We observed from simulations that matter may accrete in stable or unstable regime.

First we investigated accretion in stable regime to a star with misalignment angle $\Theta=15^{\circ}$ in two cases of small and tiny magnetosphere. We observed that small magnetosphere formed
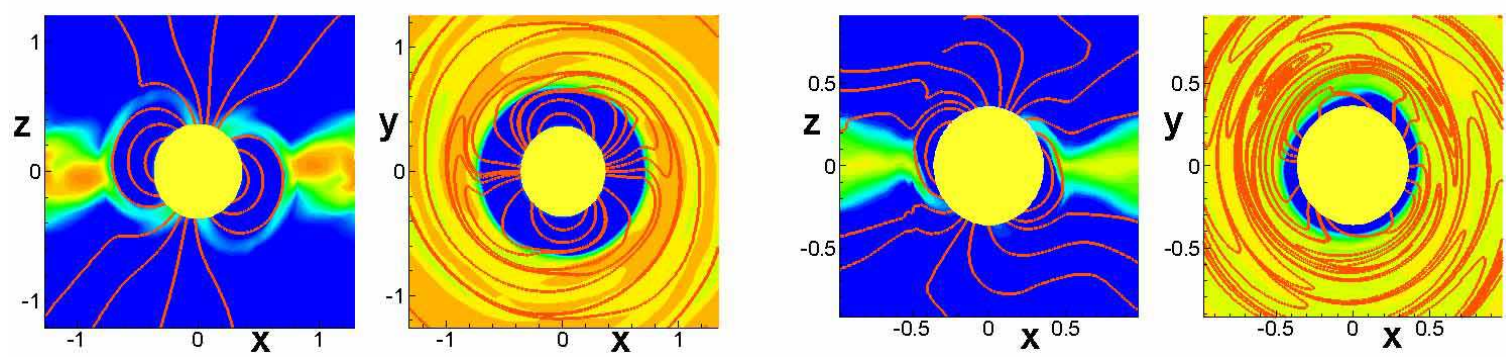

Fig. 2.- 3D MHD simulations of accretion through the magnetic boundary layer of a star (in the stable regime) with a misalignment angle $\Theta=15^{\circ}$. Left two panels show slices of density distribution and sample magnetic field lines in the case of a small magnetosphere. The two righthand panels show similar slices but for a tiny magnetosphere. 
and matter accreted through funnel streams (see Figure 2, left panels). In the case of about twice as smaller dipole field of the star a tiny magnetosphere formed and also disrupted the disk (see Figure 2, right panels). Equatorial slice shows that the magnetic field lines are wrapped by the disk and may influence its structure. In particular, non-axisymmetric bending-type waves have been observed in the inner parts of the disk.

Another run at smaller misalignment angle $\Theta=5^{\circ}$ led to unstable accretion but of unusual kind: instead of sporadically forming unstable tongues as in the unstable regime (Kulkarni \& Romanova 2008a,b; Romanova, Kulkarni \& Lovelace 2008), we observed the formation of two symmetric equatorial tongues which connect the star with the disk and which rotate around the star with angular velocity of the inner disk (see Figure 3). The tongues form two equatorial spots which rotate faster than the star: each spot rotates about 4 times faster than the star. The frequency approximately corresponds to the frequency of the inner disk and will change when position of the inner disk change. Thus a star in this accretion regime will reveal high frequency QPOs with frequency drift determined by the accretion rate to the star. Such frequency drift and correlation with matter flux is observed in AMXPs (e.g., van der Klis 2000).

C and D. UNSTABLE AND STABLE REGIMES OF ACCRETION. For even lower accretion rates, the disk matter is stopped by the magnetosphere at a larger distance, a few radii of the star. Recent 3D MHD simulations have shown that in this case the matter may accrete either through symmetric funnel streams which are lifted above the magnetosphere (stable regime), or through an interchange instability, where temporary stochastic tongues of matter form at the disk-magnetosphere boundary and penetrate deeply into magnetosphere. These tongues form multiple hot spots on the surface of the star. Figure 4 shows both regimes of accretion and the corresponding light-curves associated with the hot spots on the surface of the star.

Stable Regime of Accretion (Funnel Streams). In the stable regime, matter accretes in two ordered funnel streams (Figure 4, left panel, see 3D MHD simulations by Romanova et al. 2003, 2004a; Kulkarni \& Romanova 2005). These two funnels form two ordered spots at the surface of the star, which vary only slightly around their preferred positions, so that the stellar period is clearly
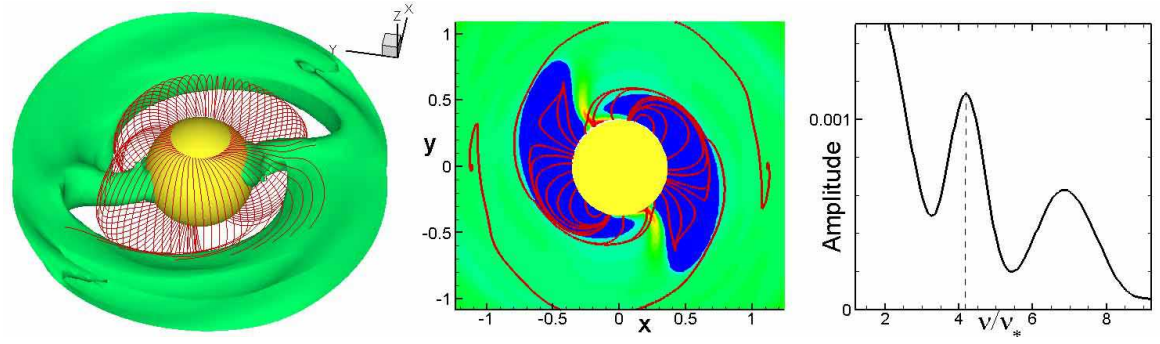

Fig. 3.- 3D MHD simulations of accretion through an unusual magnetic boundary layer which formed in the case of a small misalignment angle $\Theta=5^{\circ}$. From left to right: 3D image of the accretion disk; an equatorial slice of the density distribution, and the Fourier power spectrum. 


\begin{tabular}{llll}
\hline & Type & $\dot{M}$ (in $M_{\odot} / y r$ ) & Type of Variability \\
\hline$r_{m}<R_{*}$ & Boundary Layer & $\dot{M}>7.3 \times 10^{-8}$ & QPO \\
$R_{*}<r_{m}<2 R_{*}$ & Magnetic BL & $6.5 \times 10^{-9}<\dot{M}<7.3 \times 10^{-8}$ & QPO ( $P_{*}$ ) \\
$2 R_{*}<r_{m}<3.1 R_{*}$ & Funnel, Instabilities & $1.4 \times 10^{-9}<\dot{M}<6.5 \times 10^{-9}$ & $P_{*}$, QPO \\
$3.1 R_{*}<r_{m}<12 R_{*}$ & "Propeller" & $1.3 \times 10^{-11}<\dot{M}<1.4 \times 10^{-9}$ & QPO ( $\left.P_{*}\right)$ \\
$r_{m}>12 R_{*}$ & Pulsar & $\dot{M}<1.3 \times 10^{-11}$ & radio $P_{*}$ \\
\hline
\end{tabular}

Table 1: Ranges of magnetospheric radii, accretion rates and expected variability features in different regimes of accretion for a neutron star with $P_{*}=2.5 \mathrm{~ms}$.
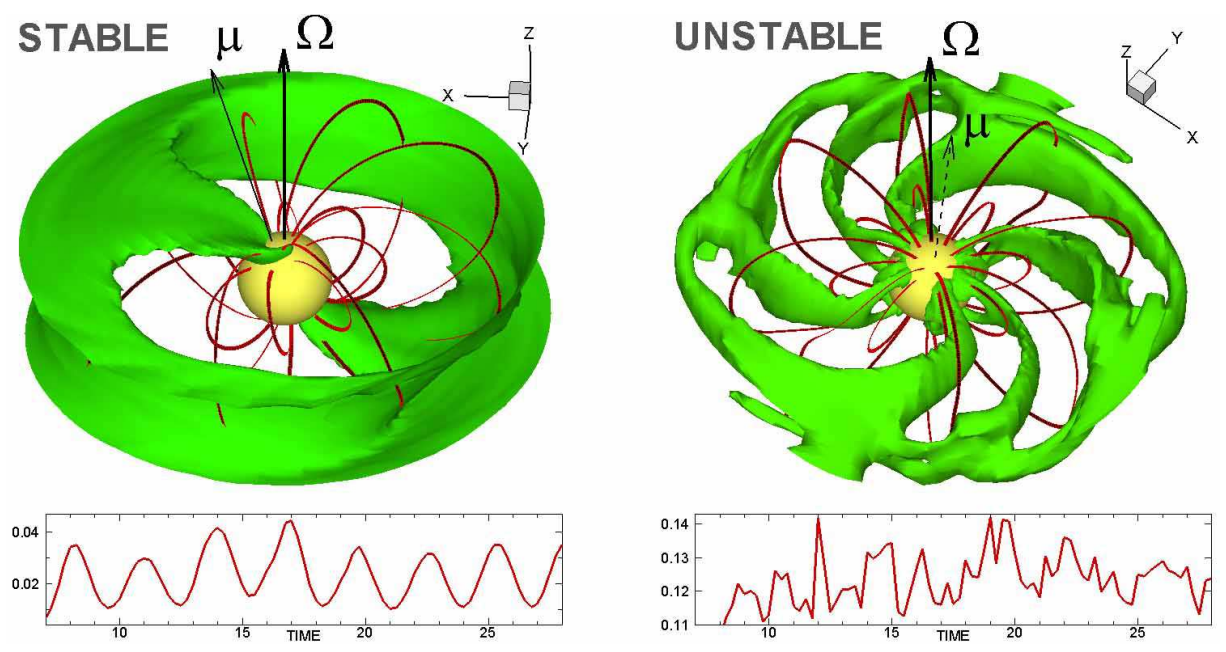

Fig. 4.- Stable (left) and unstable (right) regimes of accretion. The bottom panels show lightcurves from the hot spots (from Romanova, Kulkarni \& Lovelace 2008). 
observed in the Fourier power spectrum. This is the regime where X-ray pulsations in AMXPs are expected. If the magnetic field is more complex than the dipole, then the hot spots have a more complex structure. However, spots will have a definite preferred locations so that a definite periodicity is expected (Long et al. 2008). Our simulations shown that a star may spin up or down due to interaction of the magnetosphere with the disk, so that the star's period is expected to wander around the rotational equilibrium state, which corresponds to the condition $r_{m} \approx 1.2 r_{c o r}$, which has been derived from simulations by Long et al. (2005) (see also Ghosh \& Lamb 1979). Such variations of the period have been recently observed, e.g., by Burderi et al. (2007) and Papitto et al. (2008). In addition to periodic variations associated with the hot spots, QPOs associated with oscillations of the inner disk regions are expected (Lovelace \& Romanova 2007; Lai \& Zhang 2008).

Simulations have shown that at small misalignment angles $\Theta<5^{\circ}$ a hot spot may rotate faster or slower than the star depending on the accretion rate (Romanova et al. 2003; Romanova et al. 2006). This may lead to the rapid phase variations and other interesting phenomena observed in AMXPs (Lamb et al. 2008a,b).

The Unstable Regime of Accretion has been observed in multiple 3D MHD simulations in cases where the misalignment angle of the dipole is small, $\Theta=5^{\circ}$ (Kulkarni \& Romanova 2008a,b; Romanova et al. 2008). In the unstable regime, matter accretes to the star through several "tongues" which form at the inner edge of the disk and accrete to the surface of the star on the dynamical time-scale, forming temporary hot spots on its surface. The light-curve from the hot spots looks stochastic (see Figure 4, right panel), and in many cases no period is detected in the wavelet or Fourier spectra. Thus, in the strongly unstable regime, a star with a full-sized magnetosphere may be in the stochastic regime of accretion. In less strongly unstable cases, both tongues and funnels are present, and periodicity can be detected.

Boundary Between Stable and Unstable Regimes: We observed from numerous simulations that (1) the stable regime usually dominates at high misalignment angles, $\Theta \gtrsim 30^{\circ}$; (2) enhancement of the accretion rate usually leads to the onset of the unstable regime (Kulkarni \& Romanova, these Proceedings). The last condition can be interpreted as follows: for a NS with a given period of rotation $P_{*}$, the growth of the accretion rate leads the gravitational force to dominate over the centrifugal (or, $r_{m}<<r_{c o r}$ ), and the instability appears more easily. At smaller accretion rates, the magnetospheric radius moves out, closer to $r_{c o r}$, and the centrifugal force becomes strong enough to oppose instability. Detailed comparisons of stable/unstable regimes with instability criteria were also performed (Kulkarni \& Romanova 2008a; Romanova et al. 2008). From other side we observed that at larger accretion rate the density of matter at the inner edge of the disk is larger which is favorable for the onset of instability.

F. "PROPELLER" REGIME. At even smaller accretion rates, the magnetospheric radius $r_{m}$ becomes larger than the corotation radius $r_{\text {cor }}$, and the star enters the "propeller" regime. Axisymmetric simulations performed in our group have shown that propeller regime may be either strong or weak. 

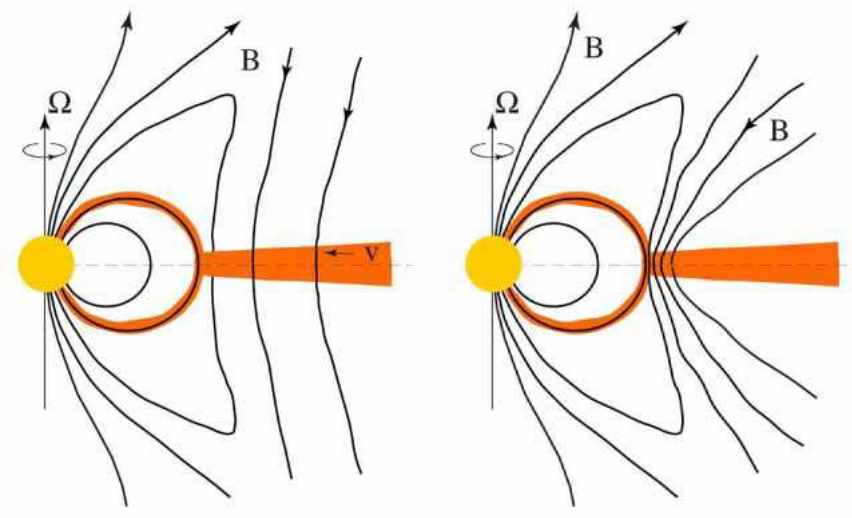

Fig. 5.- Disk-magnetosphere interaction for different magnetic Prandtl numbers. The left-hand panel is for $\operatorname{Pr}=1$, when the disk matter flows in and penetrates through the magnetosphere diffusively at the same rate. The right-hand panel is for $\operatorname{Pr}>>1$, where the radial accretion speed is significantly larger than the diffusion speed of the field through the matter.
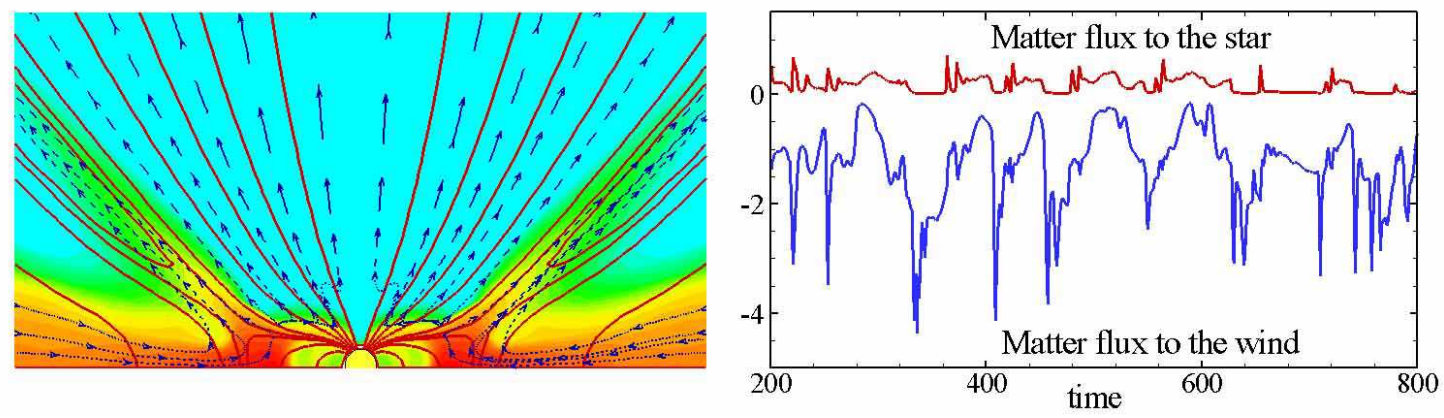

Fig. 6.- The left panel shows outflow of matter (background, selected field lines (red) and streamlines (blue) in the strong propeller regime. The fluxes at the right panel show that the process of accretion and outflows is episodic (from Romanova et al. 2005). 
In the Strong Propeller Regime, disk matter acquires angular momentum from the rotating magnetosphere fast enough that most of it is ejected in conical outflows. At the same time a significant amount of angular momentum and energy flows along the open stellar field lines, giving an axiallydirected Poynting-flux dominated jet (Romanova et al. 2005; Ustyugova et al. 2006). Multiple simulations have shown that the strong propeller operates when $\operatorname{Pr}>>1$. In this case the field lines are bunched into an X-type point (see Figure 5, right panel) which is favorable for outflows, as discussed by Shu and collaborators (e.g., Cai et al. 2008). In this regime, the cycles of matter accumulation at the inner disk, its diffusion through magnetosphere and outbursts to jets are observed with some accretion to the star (see also Goodson et al. 1997). Smaller ratios $r_{m} / r_{c o r}$ and higher diffusivities (still at $\mathrm{Pr} \gg 1$ ) are also favorable for the strong propeller regime. Oscillations are often quasi-periodic, with frequency $\nu_{Q P O}=0.01-0.2 \nu_{*}$, where the lowest frequency is observed at the lowest diffusivity, and vice-versa.

In the Weak Propeller Regime, when $\operatorname{Pr} \approx 1$, the magnetosphere interacts with the disk, and the star loses its angular momentum without outflows. The disk may oscillate due to such interaction, and most of the incoming disk matter may accrete to the star's surface (Romanova et al. 2004b). It is often the case that the rotating magnetosphere (modified by the azimuthal wrapping of the field) pushes the disk to larger distances, and matter may accrete from that point. In the limit of a very weak propeller where $r_{m} \approx r_{c o r}$, stellar pulsations and different QPO frequencies are expected as in the case of funnel flow accretion. If magnetosphere is large enough then accretion may be stopped by the centrifugal barrier of the fast rotating magnetosphere in both "propeller" regimes.

G. PULSAR REGIME. For even smaller accretion rates, the radius of the magnetosphere becomes larger than the light cylinder radius, $r_{L}=c / \Omega_{*}$. In this regime, accretion to the surface of the NS is suppressed, and the star becomes a radio-pulsar. This stage is least investigated numerically and it is not clear, for example, at what values of $r_{m} / r_{L C}$ the radio-pulsar emission dominates.

\section{Discussion}

Analysis of different stages of evolution of accreting magnetized neutron stars have shown that they have different appearances for different accretion rates. In addition, their appearance may be different depending on other factors, such as the magnetic diffusivity and the Prandtl number (ratio of viscosity to diffusivity). We chose as an example a typical NS with period $P_{*}=2.5 \mathrm{~ms}$ $\left(\nu_{*}=400 \mathrm{~Hz}\right.$ ), and derived accretion rates from equation (2) corresponding to the boundaries between different regimes. The results are shown in the Table. As we see from simulations of different regimes, periodic oscillations (with the star's period) are observed only in the regime of magnetospheric accretion which corresponds to a rather narrow range of accretion rates, $1.4 \times$ $10^{-9} M_{\odot} / y r<\dot{M}<6.5 \times 10^{-9} M_{\odot} / y r$. This fact may explain the appearance of pulsations in intermittent AMXPs in a narrow range of $\dot{M}$ as discussed by Altamirano et al. (2008) in the case of SAX J1748.9-2021. If the pulsations are also present in the magnetic boundary layer case and in 
the weak propeller regime, then these boundaries for $\dot{M}$ will be somewhat wider.

\section{REFERENCES}

Altamirano, D., Casella, P., Patruno, A., Wijnands, R., van der Klis, M., 2008, ApJ, 674L, 45A

Arons, J., Lea, S. M., 1976, ApJ, 207, 914

Bessolaz, N., Zanni, C., Ferreira, J., et al., 2008, A\&A, 478, 155

Burderi, L., Di Salvo, T., Lavagetto, G., et al., 2007, ApJ, 657, 961

Cai, M. J., Shang, H., Lin, H.-H., Shu, F.H., 2008, ApJ, 672, 489

Casella, P., Altamirano, D., Patruno, A., Wijnands, R., van der Klis, M., 2008, ApJ, 674L, 41C

Cumming, A., Zweibel, E., Bildsten, L. 2001, ApJ, 557, 958

Elsner, R. F., Lamb, F. K., 1977, ApJ, 215, 897

Fisker, J.L., Balsara, D.S., 2005, ApJ, 635, L69

Ghosh, P., Lamb, F. K., 1979, ApJ, 232, 259

Goodson, A.P, Winglee, R.M., Boehm, K.-H., 1997, 489, 199

Kulkarni, A. K., Romanova, M. M., 2005, ApJ, 633, 349

Kulkarni, A. K., Romanova, M. M., 2008a, MNRAS, 386, 673

Kulkarni, A. K., Romanova, M. M., 2008b, MNRAS, submitted, 2008arXiv0812.0241K

Lai, D., Zhang, H., 2008, ApJ, 683, 949

Lamb, F.K., Miller, M.C., 2001, ApJ, 554, 1210

Lamb, F.K., Boutloukos, S., Van Wassenhove, S., Chamberlain, R.T., Lo, K.H., Miller, M.C. 2008a, ApJ Letters, in press, 2008arXiv0809.4016L

Lamb, F.K., Boutloukos, S., Van Wassenhove, S., Chamberlain, R.T., Lo, K.H.; Clare, A., Yu, W., Miller, M.C. 2008b, ApJ, in press, 2008arXiv:0808.4159

Long, M., Romanova, M.M., Lovelace, R.V.E. 2005, ApJ, 634, 1214

Lovelace, R.V.E., Romanova, M.M, 2007, ApJ, 670, L13

Lovelace, R.V.E., Romanova, M.M, Bisnovatyi-Kogan, G.S. 2005, ApJ, 625, 957L 
Miller, M.C., Lamb, F.K., \& Psaltis, D. 1998, ApJ, 508, 791

Papitto, A., Menna, M. T., Burderi, L., di Salvo, T., Riggio, A., 2008, MNRAS, 383, 411

Romanova, M.M., Kulkarni, A., Long, M., Lovelace, R.V.E., Wick, J.V., Ustyugova, G.V., Koldoba, A.V. 2006, Advances in Space Research, 38, 2887

Romanova, M. M., \& Kulkarni, A. K. 2008, MNRAS (submitted), arXiv:0812.0384

Romanova, M. M., Kulkarni, A. K., \& Lovelace, R.V.E. 2008, ApJ, 673, L171

Romanova, M. M., Ustyugova, G. V., Koldoba, A. V., Lovelace, R. V. E., 2003, ApJ, 595, 1009

Romanova, M. M., Ustyugova, G. V., Koldoba, A. V., Lovelace, R. V. E., 2004a, ApJ, 610, 920

Romanova, M. M., Ustyugova, G. V., Koldoba, A. V., Lovelace, R. V. E., 2004b, ApJ, 616, L151

Romanova, M. M., Ustyugova, G. V., Koldoba, A. V., Lovelace, R. V. E., 2005, ApJ, 635, L165

van der Klis, M., 2000, ARA\&A, 38, 717

Wang, Y.-M., Robertson, J. A., 1985, ApJ, 299, 85

Ustyugova, G.V., Koldoba, A.V., Romanova, M.M., Lovelace, R.V.E. 2006, ApJ, 646, 304

Warner, B., Woudt, P.A., 2002, MNRAS, 335, 84

Wijnands, R.A.D, van der Klis, M., 1998, Nature, 394, 344 